\title{
Some Aspects of Rain Formation in Warm Cloud (II). Liquid Water Content as a Function of Upward Velocity*
}

\author{
By M. Komabayasi \\ Geophysical Institute, Tokyo University \\ (Manuscript received 17 October, 1957)
}

\begin{abstract}
The liquid water content of a precipitating warm cloud is expressed as a function of upward velocity, taking into account the difference between the rate of condensation which accumulates the liquid water in the cloud and the rate of rainfall, which carries it out of the cloud. As for the condensation nuclei of raindrops, a simplified analytical expression of Woodcock's size distribution (1953) is used.

The liquid water content in a steady state is found to be proportional to $1 / 3$ power of the upward velocity, the numerical values of which, for example, are $0.42,0.90$ and $1.93 \mathrm{~g} / \mathrm{m}^{3}$ for upward velocities $10 \mathrm{~cm} / \mathrm{sec}, 1$ and $10 \mathrm{~m} / \mathrm{sec}$ respectively in a typical condition for the cloud and sea-salt nuclei.

In transient state, the liquid water content of the cloud is found to take a maximum at a certain time after the beginning of cloud formation if upward velocities are larger than $10 \mathrm{~cm} / \mathrm{sec}$, thereafter it begins to decrease due to falling out of raindrops and approaches a value of steady state.

Preferred appropriate upward velocity for initial raindrop formation due to the presence of large sea-salt nuclei is discriminated from that due to the coalescence mechanism under the presence of various size distribution of nuclei.

Effect of the entrainment of surrounding air into cloud on the salinity of rain water is qualitatively discussed from the viewpoint of nuclei budget of the cloud.
\end{abstract}

\section{Introduction to Part II}

The liquid water content of the cloud is one of the main factors which governs the growth of raindrops in a cloud. Its change must be studied if we intend to obtain accurately the time of onset of rainfall, the intensity of rain, and the change of raindrop size spectrum with respect to time. Besides, it may be through the liquid water content as well as the supersaturation of the water vapour that upward velocity inside cloud has a relation to the growth of the precipitation element. Dynamical and thermodynamical factors of a cloud would give an influence on mechanism of precipitation through the liquid water content of the cloud.

In Part I, we estimated relations between the large sea-salt nuclei and rainfall based on the sea-salt hypothesis, where a model of cloud was used in which distances between

* Division of Meteorology, Contribution No. 111. each air parcel and the cloud base do not change with respect to time. In that calculation, the liquid water content of the cloud was assumed to be constant with respect to time. Here in Part II, we intend to treat the change in the 1.w.c. (liquid water content) with respect to time using the result obtained in Part I as a first approximation.

Generally speaking, it is considered that the 1.w.c. of a cloud could be determined from a rate of condensation, the downward transport of the cloud water by rainfall, the entrainment of surrounding air into the cloud. Stommel (1947) estimated the quantity of entrainment of the air into clouds using data on temperatures observed at all levels inside and outside trade-wind cumulus and calculated the resulting l.w.c. within the clouds. The calculated values of 1.w.c. seem to be likely to occur in nature though a direct comparison could not be made between the calculated and observed values. So the effect of entrain- 
ment may be important for the determination of the 1.w.c., especially in small cumulus surrounded by dry air, which shows no precipitation. But, the quantitative estimate of the lateral mixing is generally very difficult at present. As the first step of the problem, here in Part II, we shall discuss the effect of rainfall on the 1.w.c. ignoring the lateral mixing. The effect of upper mixing is taken into consideration. Accordingly the consideration made in the present work is confined to phenomena well inside large cloud region where the effect of lateral mixing may be expected to be less pronounced as stated by Ludlam and Scorer (1953). Qualitative discussion on the lateral mixing will be made a little at the last part of section 5 .

\section{The fundamental equation determining the liquid water content}

The change of the liquid water content $M$ in an individual air parcel with respect to time $t$ can be expressed as

$$
\frac{d M}{d t}=m+\frac{\partial G}{\partial z}
$$

where $m$ is the rate of condensation and $G$ the downward mass flux of liquid water due to gravitational falling at a height $z$ above the cloud base, $\frac{\partial G}{\partial z}$ being mass convergence of the liquid water. Integrating this with respect to $z$ from the base to the top of a cloud column, we obtain

$$
\begin{gathered}
h \frac{d \bar{M}}{d t}=h \bar{m}+G(h)-G(0), \\
\bar{M}=\frac{1}{h} \int_{0}^{h} M d h, \quad \bar{m}=\frac{1}{h} \int_{0}^{h} m d h,
\end{gathered}
$$

where $h$ is the cloud thickness, $\bar{M}$ and $\bar{m}$ are an average, 1.w.c. and an average rate of condensation in the cloud column. $G(h)$ is the downward transport of the liquid water at the top of the cloud column, that is, the transport due to the mixing at the upper boundary of the cloud. In general, $G(h)$ is of negative value. Below we use $G=-G(h)$ instead. $G(0)$ is the downward transport of the liquid water at the cloud base, in other word, the intensity of rainfall. Accordingly, equation (2) becomes

$$
h \frac{d \bar{M}}{d t}=(h \bar{m}-G)-\rho_{w} W
$$

where $\rho_{w}$ is the density of liquid water and $W$ is the rainfall intensity expressed in a rate of increase of thickness of liquid water. $W$ is determined from the size distribution of raindrops. Raindrop size distribution is again determined from the size distribution of the nuclei, 1.w.c. and the thickness of the cloud, and as discussed in detail in Part I, from the pattern of the upward current.

Here we adopted the same model of cloud as used in Part I; the air column upglides parallel to a slope of the surface of the cloud base, so distances between each part of the air column and the base of the column do not change with respect to time. All raindrops are assumed to form on large sea-salt nuclei. The characteristic point of this model is that in this cloud larger raindrops are to form on larger nuclei. Then, we can use results of calculation obtained in Part I.

To solve equation (3), we use as a first approximation the relation (14) of Part I which shows the relation between the rainfall intensity and the size distribution of large sea-salt nuclei.

$$
\begin{aligned}
W(t)=\frac{4 \pi}{3} \alpha r_{0}^{4} \exp \left(\frac{E \alpha M}{\rho_{w}} t\right) \int_{\nu_{2}}^{\nu_{1}} f(\nu) \\
\\
\times \exp \left(-\frac{E \alpha a}{\rho_{w}} \frac{M}{\nu}\right) d \nu
\end{aligned}
$$

where $W(t)$ is the rainfall intensity at a time $t$ after the beginning of condensation of the cloud column considered, $M$ is the l.w.c. of the cloud, $\alpha, r_{0}, E$, and $a$ are positive numerical constants respectively, $f(\nu) d \nu$ the number concentration of the large sea-salt particles with mass between $\nu$ and $\nu+d \nu$ contained in the air before the condensation begins, $\nu_{1}$ the mass of nucleus which at time $t$ just reaches a critical radius, drops smaller than it being assumed not to fall appreciably by gravity, $\nu_{2}$ the upper limit of the mass of the large sea-salt nuclei which are present at the time $t$ in the cloud column. As discussed in Part I, $\nu_{1}$ and $\nu_{2}$ are functions of time respectively,

$$
\left.\begin{array}{l}
\nu_{1}=\frac{a}{t}, \\
\nu_{2}=\nu_{\max } \quad \text { for } t \leqq t^{*},
\end{array}\right\}
$$




$$
\begin{aligned}
& \nu_{2}=a\left\{t-\frac{4 \rho_{w}}{E \alpha M} \ln \left(\frac{E M}{4 \rho_{w} r_{0}} h+1\right)\right\}^{-1} \\
& t^{*}=\frac{a}{\nu_{\max }}+\frac{4 \rho_{w}}{E \alpha M} \ln \left(\frac{E M}{4 \rho_{w} r_{0}} h+1\right),
\end{aligned}
$$

where $\nu_{\max }$ is the mass of largest nuclei of the air at $t=0$, that is $f(\nu)=0$ for $\nu>\nu_{\max }$, and $t^{*}$ is the time before which nuclei of $\nu_{\max }$ still remain in the cloud column. After $t^{*}$ elapses, they entirely fall out of the cloud and the upper limit of the mass of nuclei present in the cloud decreases successively with increasing time. If we put (4) into (3) and substitute $\bar{M}$ for $M$, we obtain

$$
\begin{aligned}
h \frac{d \bar{M}}{d t}= & (h \bar{m}-G) \\
& -\rho_{w} \frac{4 \pi}{3} \alpha r_{0}^{4} \exp \left(\frac{E \alpha \bar{M}}{\rho_{w}} t\right) \\
& \times \int_{\nu_{1}}^{\nu_{2}} f(\nu) \exp \left(-\frac{E \alpha a}{\rho_{w}} \frac{\bar{M}}{\nu}\right) d \nu,
\end{aligned}
$$

which is the equation relating the 1.w.c. to the rate of condensation $\bar{m}$, cloud thickness $h$, and the size distribution of large sea-salt nuclei $f(\nu)$. In order to research analytically the nature of $\bar{M}$ with respect to time, we express the size distribution of nuclei as follows,

$$
\left.\begin{array}{ccc}
f(\nu)=\frac{A}{\nu^{2}} & \text { for } \quad \nu \leqq \nu_{\max } \\
f(\nu)=0 & \text { for } \quad \nu>\nu_{\max }
\end{array}\right\}
$$

As discussed in Part $I$, this expression is equivalent to Junge's inverse third power law of distribution for aerosols (1953) and can represent approximately Woodcock's (1953) size distributions of large sea-salt nuclei by choosing appropriate numerical valus of $A$ and $\nu_{\max }$.

If we put (7) into (4), $W(t)$ becomes

$$
\begin{aligned}
& W(t)=\rho_{w} \frac{4 \pi r_{0}^{4}}{3 a E} \frac{A}{\bar{M}} \exp \left(\frac{E \alpha \bar{M}}{\rho_{w}} t\right) \\
& \quad \times\left\{\exp \left(-\frac{E \alpha a}{\rho_{w}} \frac{\bar{M}}{\nu_{2}}\right)-\exp \left(-\frac{E \alpha a}{\rho_{w}} \frac{\bar{M}}{\nu_{1}}\right)\right\} .
\end{aligned}
$$

Using (5),

$$
W(t)=0 \quad \text { for } t \leqq \frac{a}{\nu_{\max }}
$$

$$
\begin{array}{r}
W(t) \\
=\rho_{w} \frac{4 \pi r_{0}^{4}}{3 a E} \frac{A}{\bar{M}}\left[\exp \left\{\frac{E \alpha \bar{M}}{\rho_{w}}\left(t-\frac{a}{\nu_{\max }}\right)\right\}-1\right] \\
\text { for } \frac{a}{\nu_{\max }}<t \leq t^{*}
\end{array}
$$

and

$$
W(t) \simeq \frac{\pi A}{192 a}\left(\frac{E \bar{M}}{\rho_{w}}\right)^{3} h^{4} \quad \text { for } t>t^{*},
$$

(10) being discussed in (16) of Part I. Using (8), (9) and (10) with equation (3), we shall discuss the change of the 1.w.c. $\bar{M}$ in the following sections.

\section{Liquid water content in a steady state}

Generally speaking, the larger the l.w.c. is, the more intensely rain falls. From (10), it can be seen that the rainfall intensity is proportional to cube of l.w.c. after $t^{*}$ elapses. So a steady state can exist where the rate of condensation balances the rainfall intensity. Putting $d \bar{M} / d t=0$ in (3), using (10)

$$
\left.\begin{array}{l}
\bar{M}=\left(\frac{192 \rho_{w}^{2} a}{\pi}\right)^{1 / 3}\left(\frac{m^{\prime}}{A}\right)^{1 / 3} \frac{1}{E h}, \\
m^{\prime}=\bar{m}-\frac{G}{h},
\end{array}\right\}
$$

is obtained as a value of the l.w.c. in a steady state. $m^{\prime}$ is an apparent rate of condensation which is reduced by upper mixing from the true rate $m$. (11) means that the average 1.w.c. will be proportional to $1 / 3$ power of the apparent rate of condensation, inversely proportional to the cloud thickness, and to $1 / 3$ power of $A$ which is a numerical value concerning the number concentration of large sea-salt nuclei. Since the rate of condensation can be assumed to increase proportionally to an upward velocity, the average 1.w.c. $\bar{M}$ can be said to be nearly proportional to $1 / 3$ power of the upward velocity of the air colum unless the cloud is so thin that $\bar{m}$ is hardly larger than $G / h$. It might seem somewhat strange that $\bar{M}$ is inversely proportional to $h$. So far as the present approximate treatment is concerned, this might be explained in the following way; in a thicker cloud the liquid water would be took away more efficiently by a larger number of falling raindrops, so that the average 1,w.c. would be reduced to a smaller 
value, at least in a steady state, thus integrated 1.w.c. from the base to the top of the cloud column $\bar{M} h$ might be rather independent on the cloud thickness. This may lead to that if the thicker cloud were found to have the larger l.w.c., this might be expected partly because the upward current would be more intense in the thicker cloud, and partly because the 1.w.c. would not stay in a steady state when the measurement is made.

On the other hand, in non-precipitating cloud a steady state of 1.w.c. occurs when $\bar{m} h=G$ is satisfied. The rate of depletion of $M$ due to upper mixing may be expected to be proportional to $M$, that is, $G \propto M$. In this case the 1.w.c. of steady state would be proportional to the upward velocity and the cloud thickness.

To obtain numerical values of (11) at various upward velocities and for different size distributions of large sea-salt nuclei, we choose $A=1.4 \times 10^{-13}, 1.15 \times 10^{-12}$ and $3.7 \times 10^{-11}$ $\mathrm{g} / \mathrm{cm}^{3}$ whereby (7) expresses approximately the average size distributions of large seasalt nuclei measured at cloud levels by Woodcock (1953) in the cases of surface wind force 1,4 and 12 respectively. In the former two cases $A / \nu^{2}$ can express rather accurately the measured distributions, where the concentrations of the nuclei with masses larger than $10^{-11} \mathrm{~g}$ are $1.4 \times 10^{4}, 1.15 \times 10^{5}$ nuclei $/ \mathrm{m}^{3}$ respectively. But in the last case for surface wind force 12 , the measured size distribution of the nuclei can not be represented well by $A / \nu^{2}$, so the numerical values of $\bar{M}$ obtained for this case are relatively inaccurate, but they are illustrated for comparison with the former two cases of lighter wind. In Table 1, the average l.w.c. $\bar{M}$ in a steady state are tabulated for different conditions. In these calculations, the average rate of condensation $\bar{m}$ in (11) was determined from the moist adiabatic process in constant upward velocities at temperatures between $15^{\circ} \mathrm{C}$ and $0^{\circ} \mathrm{C}$ for pressures between $850 \mathrm{mb}$ and $500 \mathrm{mb}$. We can not estimate $G$, so here $G / h$ is assumed to be negligibly small as compared with $\bar{m}$. Perhaps this neglection may be allowed for the case $h>1000 \mathrm{~m}$. But, if we obtain the value of $G$, we can use Table 1 by adopting an effective upward velocity which is $m^{\prime} / \bar{m}$ $=1-(G / \bar{m} h) \simeq 1-(G / W)$ times the true upward
Table 1. Liquid water content in a steady state as a function of upward velocity

\begin{tabular}{|c|c|c|c|c|}
\hline \multirow{3}{*}{$\begin{array}{l}\text { Cloud } \\
\text { thickness }\end{array}$} & \multirow{3}{*}{$\begin{array}{l}\text { Upward } \\
\text { velocity }\end{array}$} & \multicolumn{3}{|c|}{ 1.w.c. of cloud in $\mathrm{g} / \mathrm{m}^{3}$} \\
\hline & & \multicolumn{3}{|c|}{ Nuclei for wind force } \\
\hline & & 1 & 4 & 12 \\
\hline \multirow{10}{*}{$1000 \mathrm{~m}$} & $1 \mathrm{~cm} / \mathrm{s}$ & 0.78 & 0.39 & 0.12 \\
\hline & 2 & 1.00 & 0.49 & 0.15 \\
\hline & 5 & 1.38 & 0.62 & 0.21 \\
\hline & 10 & 1.72 & 0.84 & 0.26 \\
\hline & 20 & 2.20 & 1.05 & 0.33 \\
\hline & 50 & 2.97 & 1.42 & 0.45 \\
\hline & $1 \mathrm{~m} / \mathrm{s}$ & 3.64 & 1.80 & 0.57 \\
\hline & 2 & 4.70 & 2.49 & 0.71 \\
\hline & 5 & 6.40 & 3.02 & 0.97 \\
\hline & 10 & 7.83 & 3.87 & 1.22 \\
\hline \multirow{10}{*}{$2000 \mathrm{~m}$} & $1 \mathrm{~cm} / \mathrm{s}$ & 0.39 & 0.19 & 0.06 \\
\hline & 2 & 0.50 & 0.24 & 0.08 \\
\hline & 5 & 0.68 & 0.33 & 0.10 \\
\hline & 10 & 0.84 & 0.42 & 0.13 \\
\hline & 20 & 1.07 & 0.52 & 0.17 \\
\hline & 50 & 1.45 & 0.71 & 0.22 \\
\hline & $1 \mathrm{~m} / \mathrm{s}$ & 1.82 & 0.90 & 0.28 \\
\hline & 2 & 2.60 & 1.13 & 0.36 \\
\hline & 5 & 3.12 & 1.54 & 0.48 \\
\hline & 10 & 3.92 & 1.93 & 0.61 \\
\hline \multirow{10}{*}{$3000 \mathrm{~m}$} & $1 \mathrm{~cm} / \mathrm{s}$ & 0.26 & 0.13 & 0.04 \\
\hline & 2 & 0.33 & 0.16 & 0.05 \\
\hline & 5 & 0.45 & 0.23 & 0.07 \\
\hline & 10 & 0.56 & 0.28 & 0.09 \\
\hline & 20 & 0.71 & 0.34 & 0.11 \\
\hline & 50 & 0.97 & 0.47 & 0.15 \\
\hline & $1 \mathrm{~m} / \mathrm{s}$ & 1.21 & 0.60 & 0.19 \\
\hline & 2 & 1.54 & 0.71 & 0.24 \\
\hline & 5 & 2.10 & 0.97 & 0.33 \\
\hline & 10 & 2.62 & 1.29 & 0.41 \\
\hline
\end{tabular}

velocity. $G$ is the evaporation due to upper mixing, $W$ the rainfall intensity in a steady state. It can be seen from Table 1 that $\bar{M}$ varies from a few tenths to a few $\mathrm{g} / \mathrm{m}^{3}$ for change in an upward velocity from the order of $\mathrm{cm} / \mathrm{sec}$ to the order of $\mathrm{m} / \mathrm{sec}$. These values seem not to be so different from values which may be usually expected to occur from the experience of observations, but comparisons between the tabulated values and actually observed values can not be made at present stage of the work, because it is not clear 
what an extent each cloud observed satifies the assumptions adopted in the present model of cloud. The values in Table 1 may be understood to show a quantitative tendency of the 1.w.c. expected to occur in a precipitating warm cloud layer with upgliding motion.

In Table 1 , it is seen that when the large nuclei are present in the atmosphere in extremely great number as in the case for wind force 12 the 1.w.c. will be small in a steady state. In Part I, it was shown that the rainfall intensity had an enormous value for the nuclei at this wind force if the l.w.c. was forced to be $1 \mathrm{~g} / \mathrm{m}^{3}$. This enormous intensity may be understandable from a great upward velocity such as $50 \mathrm{~m} / \mathrm{sec}$ for $h=2000 \mathrm{~m}$ and $150 \mathrm{~m} / \mathrm{sec}$ for $h=3000 \mathrm{~m}$ which would be necessary to keep $M=1 \mathrm{~g} / \mathrm{m}^{3}$ if the nuclei is the case.

Before reaching a value in a steady state, $\bar{M}$ will increase from zero to the limit monotonously for weak upward velocities, while $\bar{M}$. will reach a maximum and begin to decrease to the limit for intense upward velocities. These transient states of 1.w.c. are discussed in the next section.

\section{Transient state of the liquid water content}

In the early stage of cloud formation the l.w.c. is expected to increase continuously because rainfall can not start at all or at least can not be so intense enough to balance the rate of condensation.

In the first stage where no appreciable fall of droplets has yet started, the 1.w.c. will increase linearly with increasing time, that is, from (8)

$$
\frac{d \bar{M}}{d t}=m^{\prime} \quad \text { for } \quad t \leqq \frac{a}{\nu_{\max }} .
$$

In the second stage where some droplets begin to fall appreciably by gravity, but droplets which start at the highest level of the cloud column have not yet reached the cloud base, we obtain from (9)

$$
\begin{aligned}
h \frac{d \bar{M}}{d t}= & h m^{\prime}-\rho_{w}{ }^{2} \frac{4 \pi r_{0}^{4}}{3 a E} \frac{A}{\bar{M}} \\
& \times\left[\exp \left\{\frac{E \alpha \vec{M}}{\rho_{w}}\left(t-\frac{a}{\nu_{\max }}\right)\right\}-1\right]
\end{aligned}
$$

$$
\text { for } \frac{a}{\nu_{\max }}<t \leqq t^{*}
$$

$t^{*}$ is determined from (5.3) where $\bar{M}$ in (5.3) should be a kind of mean value of $\bar{M}$ with respect to time, but here for the sake of simplicity substituted by the value of $\bar{M}$ in a steady state obtained from (11).

When the largest nuclei of mass $\nu_{\max }$ which were initially suspended at the top of the cloud column reach the base by their falling, the second stage ends and the third begins. In this stage the upper limit of size spectrum of nuclei continues to decrease as nuclei rain out: Equation (3) becomes by use of (10)

$$
\frac{d \bar{M}}{d t}=m^{\prime}-\frac{\pi E^{3} A}{192 \rho_{w}^{2} a} h^{3} \bar{M}^{3} .
$$

In Fig. 1, by numerical integration of (12), (13) and (14), the changes of $\bar{M}$ with respect to time are illustrated for various upward

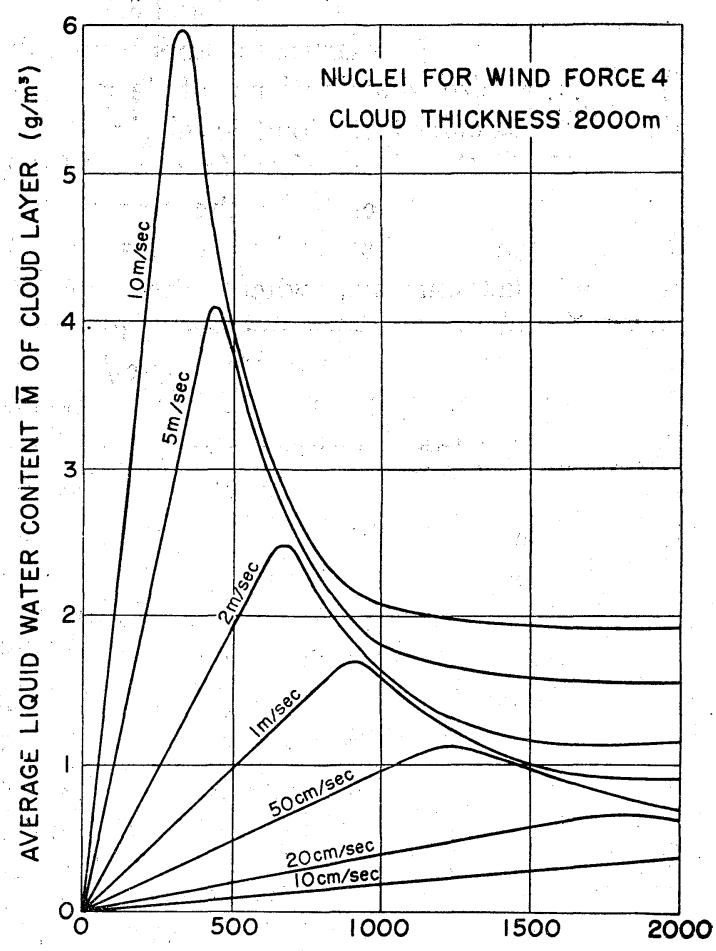

TIME AFTER BEGINNING OF CONDENSATION (sec)

Fig. 1. Change of 1.w.c. with respect to time. velocities in a cloud with $2000 \mathrm{~m}$ thickness and sea-salt nuclei for wind force 4 ((14) can be easily solved analytically). For upward velocities larger than $10 \mathrm{~cm} / \mathrm{sec} \bar{M}$ can be seen 
to increase at the beginning nearly straight to a maximum, then to decrease and approach to a value in a steady state. On the other hand, for upward velocities smaller than $10 \mathrm{~cm} / \mathrm{sec}$, though they are not illustrated fully in Fig. 1, $\bar{M}$ is found to increase monotonously to a limit. This means that when upward currents are intense, the large l.w.c. accumulates rapidly before raindrops grow large enough to fall so intensely as to balance the rate of condensation, and after the rainfall begins the intense rain reduces the 1.w.c., then rainfall becomes to weaken due to a decrease of 1.w.c. and approaches a steady state. Thus $\bar{M}$ reaches a maximum a short time before rain falls intensely. On the other hand, when upward currents are weak it is

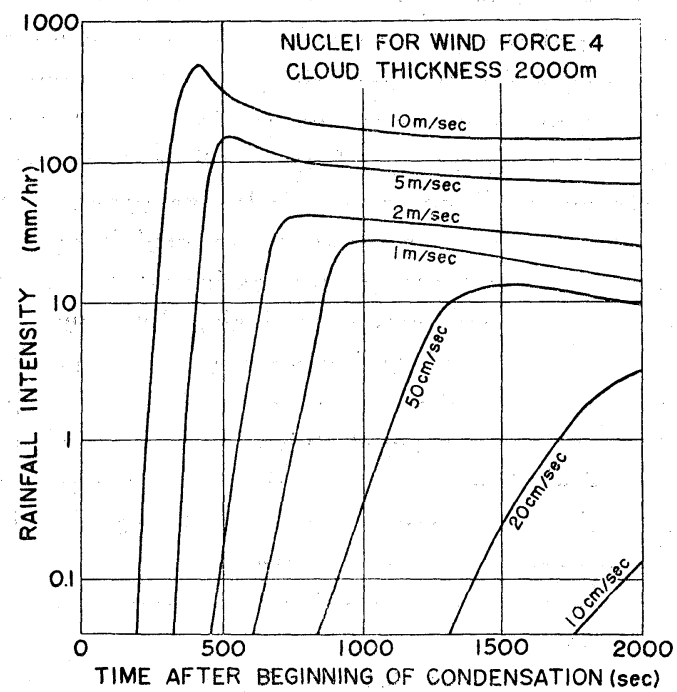

Fig. 2. Change of rainfall intensity with respect to time.

considered that the 1.w.c. has not a sufficient time to reach a large value before raindrops mature, so it reaches monotonously a steady state.

From Fig. 1, it can be seen that the more intense the upward current is, the sooner a maximum of $\bar{M}$ appears and the larger its value is. Corresponding to this change of 1.w.c., rainfall intensities are also found to have a similar change with respect to time. In Fig. 2, calculated rainfall intensities $W(t)$ corresponding to each curve of Fig. 1 are illustrated. The rainfall intensity can be seen to increase rather suddenly and reach a maximum a few hundred seconds after the 1.w.c. reached its own maximum. The weaker the upward current is, the longer this time lag is found to become. When the upward currents are weaker than $10 \mathrm{~cm} / \mathrm{sec}$, the rainfall intensity has no maximum. Here, it should be noticed that the consideration is made on individual air parcels. Consequently, $W(t)$ can not always correspond to a change of rainfall intensity observable at an observation site fixed to the ground.

Table 2. Maximum of 1.w.c. as a function of upward velocity and mass of largest nuclei. For example, ${ }_{(879)}^{1.68}$ represents that maximum of 1.w.c. appears at a time $879 \mathrm{sec}$ after beginning of condensation and the value of the maximum is $1.68 \mathrm{~g} / \mathrm{m}^{3} .0 .33^{*}$ represents that 1.w.c. approaches $0.33 \mathrm{~g} / \mathrm{m}^{3}$ monotonously.

\begin{tabular}{|c|c|c|c|c|c|}
\hline \multirow{2}{*}{$\begin{array}{l}\text { Upward } \\
\text { velocity }\end{array}$} & \multicolumn{5}{|c|}{ Mass of largest nuclei in $\mathrm{g}$} \\
\hline & $10^{-11}$ & $10^{-10}$ & $10^{-9}$ & $10^{-8}$ & $10^{-7}$ \\
\hline $1 \mathrm{~cm} / \mathrm{s}$ & $0.19 *$ & $0.19^{*}$ & $0.19^{*}$ & $0.19 *$ & $0.19^{*}$ \\
\hline 2 & $\begin{array}{c}0.30 \\
(7840)\end{array}$ & $0.24^{*}$ & $0.24^{*}$ & $0.24 *$ & $0.24^{*}$ \\
\hline 5 & $\begin{array}{c}0.70 \\
(7320)\end{array}$ & $0.35^{*}$ & $0.33^{*}$ & $0.33^{*}$ & $0.33^{*}$ \\
\hline $10 \mathrm{~cm} / \mathrm{s}$ & $\begin{array}{c}1.28 \\
(6700)\end{array}$ & $\begin{array}{c}0.53 \\
(2770)\end{array}$ & $\begin{array}{c}0.46 \\
(2410)\end{array}$ & $\begin{array}{c}0.45 \\
(2360)\end{array}$ & $\begin{array}{c}0.45 \\
(2360)\end{array}$ \\
\hline 20 & $\begin{array}{c}2.40 \\
(6280)\end{array}$ & $\begin{array}{c}0.70 \\
(2120)\end{array}$ & $\begin{array}{c}0.69 \\
(1830)\end{array}$ & $\begin{array}{c}0.69 \\
(1810)\end{array}$ & $\begin{array}{c}0.69 \\
(1810)\end{array}$ \\
\hline 50 & $\begin{array}{c}5.70 \\
(5970)\end{array}$ & $\begin{array}{c}1.44 \\
(1510)\end{array}$ & $\begin{array}{c}1.16 \\
(1220)\end{array}$ & $\begin{array}{c}1.13 \\
(1184)\end{array}$ & $\begin{array}{c}1.13 \\
(1184)\end{array}$ \\
\hline $1 \mathrm{~m} / \mathrm{s}$ & $\begin{array}{c}11.2 \\
(5870)\end{array}$ & $\begin{array}{c}2.33 \\
(1220)\end{array}$ & $\begin{array}{l}1.74 \\
(912)\end{array}$ & $\begin{array}{l}1.68 \\
(879)\end{array}$ & $\begin{array}{l}1.68 \\
(879)\end{array}$ \\
\hline 2 & $\begin{array}{c}22.2 \\
(5760)\end{array}$ & $\begin{array}{l}3.80 \\
(993)\end{array}$ & $\begin{array}{l}2.65 \\
(693)\end{array}$ & $\begin{array}{l}2.54 \\
(665)\end{array}$ & $\begin{array}{l}2.54 \\
(665)\end{array}$ \\
\hline 5 & $\begin{array}{c}55.0 \\
(5760)\end{array}$ & $\begin{array}{l}7.50 \\
(787)\end{array}$ & $\begin{array}{l}4.38 \\
(459)\end{array}$ & $\begin{array}{l}4.10 \\
(430)\end{array}$ & $\begin{array}{l}4.07 \\
(430)\end{array}$ \\
\hline 10 & $\begin{array}{l}110.0 \\
(5760)\end{array}$ & $\begin{array}{r}13.3 \\
(698)\end{array}$ & $\begin{array}{l}6.47 \\
(338)\end{array}$ & $\begin{array}{l}5.90 \\
(309)\end{array}$ & $\begin{array}{l}5.84 \\
(307)\end{array}$ \\
\hline
\end{tabular}

In Table 2 , maximum values of 1.w.c. are tabulated for various upward velocities and nuclei conditions. These values were calculated from following approximate solution. From (13) and also from Fig. 1, it can easily be shown that a maximum of $\bar{M}$ expressed by (13) are situated in $t-\bar{M}$ coordinates very closely to a point at which a straight line $\bar{M}=m^{\prime} t$ and a curve along which $d \bar{M} / d t$ of 
(13) $=0$ intersect each other, where the latter curve can be represented by a hyperbola-like curve,

$$
\begin{aligned}
& \bar{M}\left(t-\frac{a}{\nu_{\max }}\right) \\
= & \frac{\rho_{w}}{E \boldsymbol{\alpha}}\left\{\ln \bar{M}-\ln \left(\rho_{w^{2}} \frac{4 \pi r_{0}{ }^{4}}{3 a E}\right)+\ln \frac{m^{\prime} h}{A}\right\} .
\end{aligned}
$$

Then, the maximum of $\bar{M}$ can be obtained as

$$
\begin{aligned}
\bar{M}_{\max } & \simeq \frac{m^{\prime}}{2} \frac{a}{\nu_{\max }} \\
& \times\left\{1+\left(1+\frac{4 \rho_{w}}{E \alpha m^{\prime}} \frac{\nu_{\max }^{2}}{a^{2}} \ln \frac{3 E a m^{\prime} h \bar{M}}{\rho_{w}^{2} 4 \pi r_{0}^{4} A}\right)^{1 / 2}\right\}
\end{aligned}
$$

at

$$
t=\frac{\bar{M}_{\max }}{m^{\prime}},
$$

where $\bar{M}$ in the right side of (15) was put to be $1 \mathrm{~g} / \mathrm{m}^{3}$ at first, then $\bar{M}$ thus calculated in the left side was substituted again into the right side for recalculation of $\bar{M}$. Tabulated $\bar{M}$ 's are thus obtained second approximation. From (15), it can be seen that maximum values of 1.w.c. will depend mainly on a rate of condensation and the mass of largest nuclei. They also depend on a cloud thickness and number of nuclei, but the dependency is less. The stronger dependency of $\bar{M}$ on $\nu_{\max }$ instead of $A$ is attributed to that $\nu_{\max }$ determines the time of beginning of frequent coagulation process, consequently the time of onset of rainfall. Of course, the downward transport of liquid water at early stage depends on the number of nuclei, but the rainfall intensity increases exponentially with time before it exceeds the rate of condensation and so rapid that the downward transports of the liquid water overcome the rate of condensation at not so different times even if there is difference in $A$. Thus, the 1.w.c. attains its maximum at a time determined mainly by $\nu_{\max }$, consequently the value of maximum is determined mainly by $\nu_{\max }$ instead of $A$. On the contrary, the 1.w.c. in a steady state is determined by $A$ instead of $\nu_{\max }$.

In Table 2, numbers asterisked show the values of 1.w.c. in a steady state instead of maximum values since in those conditions where upward currents are weak the third stage (14) begins before $d \bar{M} / d t$ vanishes in the second stage (13). In this range of upward velocities and $\nu_{\max }, \bar{M}$ approaches monotonously its limit.

\section{Discussion}

Change of 1.w.c. with respect to time was derived in the previous section under an assumption that all raindrops form on large sea-salt particles. But this assumption may be wrong when the 1.w.c. increases to a large value since mechanism of drop formation other than rapid condensation on large sea-salt particles may be at work quicker or with a comparable rate. Perhaps this new mechanism may be some coalescence process which would produce droplets with radii of 20 to 30 microns from smaller cloud droplets. Discussions have been made on this growth process by many authors as were referred in Part I. If the large sea-salt particles of such sufficient number as stated by Woodcock (1953) are present in the air of water-saturation, several hundred drops per cubic meter are expected to grow by condensation alone to a size between 20 and 30 microns in radius within some tens of minutes after the cloud formation. So this new mechanism must have a rate greater than or at least comparable to a production rate by above mentioned sea-salt presence if this mechanism has a meteorological significance in a cloud containing a number of seasalt particles.

Coalescence process among small droplets of 10 to 20 microns in radius have been discussed by East (1957) and shown to aquire a sufficient production rate of droplets larger than 20 to 30 microns if the 1.w.c. has a large value. Telford (1955) discussed the transition of the spectrum of cloud droplets from the stochastical point of view, especially on the largest end of the size spectrum. This effect is considered to depend on number concentration of cloud droplets rather than on a value of 1.w.c., but large 1.w.c. is usually expected to occur in intense upward current, so the larger l.w.c. would correspond larger number of droplets since the larger ascending velocity would activate a larger number of condensation nuclei (Howell 1949). Consequently, 
both processes discussed by East and Telford may be enhanced greatly by an increase in 1.w.c.. Pearcey and Hill (1957) have shown the possibility of greater collision efficiency between small spheres of nearly equal sizes. If this is true for the coalescence of cloud droplets in the actual atmosphere, the importance of coalescence process for drop formation may extend its domain into smaller radius range than currently expected, because numerous small droplets are concentrated in a narrow size range of 20 microns or less in radius.

Here we intend to determine the following two prefered conditions of cloud; under the one of which the presence of sea-salt particles alone can induce the initiation of raindrop formation and under the other the coalescence process may participate or may determine the initiation of rain entirely. For this purpose we use the values of 1 .w.c. obtained in the previous section as a determining factor. East (1957) shows that growth of small cloud droplets would acquire a sufficient rate enough to induce rainfall if the 1.w.c. of the cloud becomes $4 \mathrm{~g} / \mathrm{m}^{3}$ or larger. So, here we adopt $4 \mathrm{~g} / \mathrm{m}^{3}$ as a critical value of $1 . w . c .$, that is, in cloud with 1.w.c. less than this critical value the coalescence process is assumed to be inactive as compared with the effect of seasalt presence.

In Fig. 3, maximum values of 1.w.c. and required time intervals for the maxima to be attained are plotted against coordinates of upward velocity and mass of largest nuclei existing. These values are taken from Table 2.

Densely shaded area shows the condition under which the maximum value of l.w.c. can not exceed the adopted critical value $4 \mathrm{~g} / \mathrm{m}^{3}$ and besides the maximum appears within $1000 \mathrm{sec}$ after the beginning of condensation. As discussed in the previous section, the time at which a maximum of 1.w.c. appears can be regarded approximately as the time of onset of substantial rainfall. Consequently, this shaded area, where upward velocities are a few $\mathrm{m} / \mathrm{sec}$ and nuclei larger than $10^{-10} \mathrm{~g}$ are present, may be considered to show most suitable condition under which rainfall would occur according rather strictly to the process described by the sea-salt hypothesis. In the present calculation, the collection efficiency of drop $E$ is assumed to be unity, and also the increase of temperature of droplets above that of the ambient air due to released heat by condensation is ignored. If we adopted Langmuir's collection efficiency and also took the temperature rise of droplets into consideration, the time required for appearance of maximum of 1.w.c. would be elongated to a value which may be about twice as large as the illustrated value. Therefore, under the condition above mentioned, rainfall would occur within about $2000 \mathrm{sec}$, that is, half an

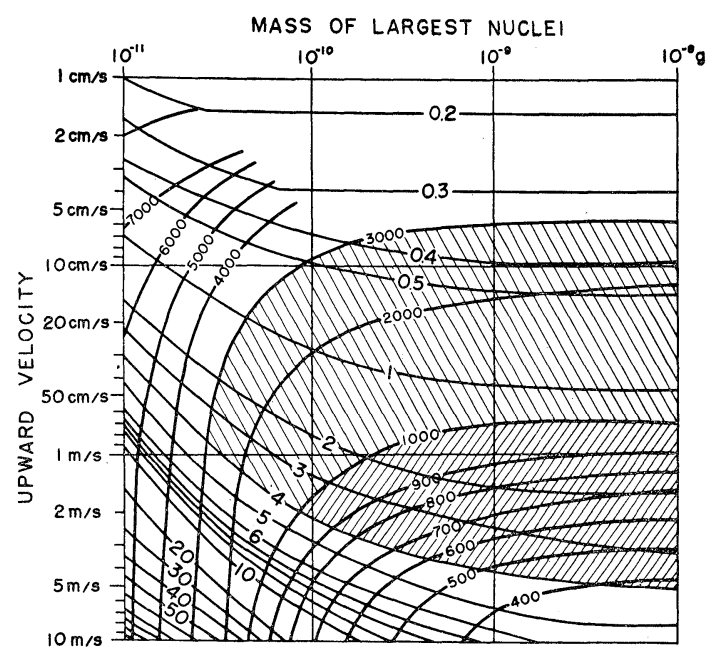

Fig. 3. Maximum of 1.w.c. and required time for its appearance. $0.4,0.5,1,2, \cdots$, represent maximum values of 1 .w.c. in $\mathrm{g} / \mathrm{m}^{3}$ and 400,500 , $\cdots, 1000, \cdots$, represent the time in sec at which the maximum of 1.w.c. appears.

hour. Ludlam (1951) estimated the condition for warm rain formation in cumulus cloud by using a cloud model whose cloud base the upward current enters steadily. It is of interest to notice that the left corner point of the densely shaded area of Fig. 3 shows the condition agreeing well with that obtained by Ludlam in spite of much difference in adopted cloud models. There may be no $a$ priori necessity that the condition derived in the present work agrees with that obtained by Ludlam. So, this agreement is considered to mean that the sea-salt nuclei of $10^{-10} \mathrm{~g}$ in weight are so large that whatever the condition of cloud may be, they can become raindrops without difficulty provided that they 
have a chance of remaining in the cloud for a period of about half an hour.

Sparsely shaded area also shows the condition under which rainfall would be possible without an operation of the coalescence process for the initiation of raindrop formation. But in this area, where upward velocities are of the order of some tens $\mathrm{cm} / \mathrm{sec}$ and sea-salt nuclei larger than $3 \times 10^{-11} \mathrm{~g}$ in weight are present, required time for onset of rainfall would be from $1000 \mathrm{sec}$ to $3000 \mathrm{sec}$. As in the former case if we corrected this value by use of Langmuir's value and effect of released heat, it would correspond from half an hour to 2 hours. Consequently there must be wide cloud area so that the individual air parcels can experience the condensation for this long time interval necessary for oneset of rainfall. If the cloud is small, the air parcel would pass across the region of upward currents before the required time elapsed and would enter the clear region or the region of downward current. In such cases rains would not occur at all or at least would become much weaker and temporary.

At further slower upward velocities, weak rainfall would be possible by the presence of large sea-salt nuclei if condensation continues very long time. So, in this case further wider cloud region than the former would be necessary for rainfall to occur.

On the other hand, at large upward velocities, the coalescence process will be able to have an important role for initiation of raindrop formation. At velocities larger than $40 \mathrm{~cm} / \mathrm{sec}$ for $\nu_{\max }=10^{-11} \mathrm{~g}, 2 \mathrm{~m} / \mathrm{sec}$ for $\nu_{\max }$ $=10^{-10} \mathrm{~g}$, and $4 \mathrm{~m} / \mathrm{sec}$ for $\nu_{\text {max }}=10^{-9} \mathrm{~g}$ respectively, the 1.w.c. of the cloud can be considered to exceed the critical value $4 \mathrm{~g} / \mathrm{m}^{3}$. So, under these conditions, the coalescence process would participate or accomplish the initiation of raindrop formation if individual air parcels can continue to ascend for the required period. But in these cases the resulting ascended distance would be so large as several kilometers since the upward velocities are great. Large vertical instability of the atmosphere would be needed for the necessary distance to be ascended in order to make rainfall.

It should be noted that discussions made above are based on the values of 1.w.c. tabulated in Table 2. These values are obtained for cloud thickness $h=2000 \mathrm{~m}$ and $A=1.15$ $\times 10^{-12}$ which is the value for the nuclei for wind force 4 . But, since the dependency of the maximum values of 1 .w.c. on cloud thickness and $A$ is not so large, it might be considered that the result of above consideration can be applied without much modification to a cloud of different thickness and different $A$. But if the nuclei has a quite different form of size distribution from $A / \nu^{2}$-law, that is, if there are some airmasses in which distribution of nuclei are quite different from those measured at trade-wind region by Woodcock (1953), the discussed result could not be applied to rain-formation in these airmass.

Finally we shall have to discuss a little about the effect of lateral mixing which we have ignored in the present work.

The effect of mixing of cloud into clear air at the top of the cloud can be taken into consideration if a value of mass flux of liquid water at the top can be aquired by observation or some theoretical consideration. If the value is obtained, we can adopt $\bar{m}-G / h$ as an average apparent rate of condensation.

On the other hand, the lateral mixing seems to be a more complicated problem. The entrained dry air may reduce the 1.w.c. of the cloud, but it may bring fresh condensation nuclei into the cloud. If there are a number of large sea-salt particles in this newly participated nuclei, size distribution of cloud droplets would be considered to receive a marked change. In this case, the calculation made in the present work can not be useful for quantitative estimation of the phenomena. But qualitatively following result might be considered, that the entrainment may reduce the intensity of rainfall, and consequently may increase the salinity of rain water, especially increase the concentration of particular substances of which large condensation nuclei consist. When the liquid water on the lateral boundary surface of the cloud evaporates by mixing, the small cloud droplets will evaporate soon and condensation nuclei of them will go out of the cloud. On the other hand, larger droplets seem to have a larger probability to survive through the evaporation 
and to fall to the ground as raindrops. So if small cloud dropletes and a larger droplets have condensation nuclei of different substances respectively, the latter substance would be concentrated in rain water as compared with the former substance. Junge $(1954,1956)$ has found that main substances of condensation nuclei are different for larger and smaller size ranges of nuclei. Since substantial part of rain water is considered to consist of the water of small cloud droplets which are captured by a relatively fewer droplets formed on large sea-salt particles, of course the concentration of the former substance could not be so small. But the more intensely the entrainment occurs, the more effectively concentrated the substance of large sea-salt nuclei would be, while the concentration of the former would not be affected so much. Therefore a ratio of concentration of the latter to that of the former in rain water may be increased by the entrainment of dry air into cloud. Inversely, from this ratio we might be possible to estimate the degree of entrainment of the precipitating cloud. About this, further investigation will be made elsewhere.

\section{Summary}

The results of the Part II may be summarized as follows.

When a cloud containing a sufficient number of large sea-salt nuclei as stated by Woodcock (1953) is ascending uniformly,

a) The average 1.w.c. of the cloud from the base to the top may increase at first to a maximum value, then begin to decrease due to onset of rainfall and approach a certain value for upward velocities larger than about $10 \mathrm{~cm} / \mathrm{sec}$. For upward velocities weaker than this, the average 1.w.c. may monotonously increase and approach a value of steady state.

b) The maximum values of 1.w.c. mainly depend on the upward velocity and the mass of the largest nuclei, while the value of 1.w.c. in a steady state depends on the number of concentration of sea-salt nuclei.

c) The 1.w.c. of a. precipitating cloud in a steady state is proportional to $1 / 3$ power of upward velocity, inversely proportional to cloud thickness and to $1 / 3$ power of the number concentration of the nuclei. Differing from this, the l.w.c. of a non-precipitating cloud may be proportional to upward velocity and to cloud thickness when the rate of evaporation due to upper mixing balances the rate of condensation.

d) For upward velocities larger than about $10 \mathrm{~cm} / \mathrm{sec}$ the substantial rainfall begins rather suddenly at about the same time when the 1.w.c. reaches its maximum. Corresponding to the change of 1.w.c., the rainfall intensity shows a maximum at its early stage. For upward velocities weaker than about $10 \mathrm{~cm} / \mathrm{sec}$, the rainfall intensity increases monotonously and approaches a value of a steady state.

e) Most suitable condition for raindrop formation due to presence of sea-salt nuclei may be considered that upward velocities are a few $\mathrm{m} / \mathrm{sec}$ and nuclei larger than $10^{-10} \mathrm{~g}$ in weight are present. In this case, the coalescence process would not be expected to play an important role for the initiation of raindrop formation since the 1.w.c. can not reach value with which the coalescence process would be fully in operation.

f) At weaker upward velocities, rain may be possible due to presence of large sea-salt nuclei, but cloud must be wider in which individual air parcels can have a sufficient time for rainformation before they go out of the region where upward current prevails.

g) At more intense upward velocities than stated in (e) or with a lack of nuclei larger than $10^{-10} \mathrm{~g}$, some coalescence mechanism may be expected to initiate the raindrop formation. In such a case, the salinity of raindrop might have no distinct relation to its size as Turner found (1955).

h) The entrainment of dry air into cloud may reduce a rainfall intensity and may increase a ratio of the concentration of substance forming large sea-salt particles to that forming small condensation nuclei in rain water.

Above discussions are based on a simplified cloud model and an approximate analytical expression of drop growth. The accurare numerical solution of the problem might be different from the above results, but it may be expected that general tendencies and roughly quantitative estimate of the phenomena could be outlined in the present work. 


\section{Acknowledgment}

The author wishes to express his deep thanks to Prof. K. Isono, Geophysical Institute, Tokyo University for his critical discussions and useful suggestions. $\mathrm{He}$ also wishes to express gratitude to Prof. S. Syōno, Geophysical Institute, Tokyo University, for his encouragement through the present work.

\section{Appendix}

List of all notations and numerical constants used in Part I and Part II are shown below. Dimensions are expressed for each of them in c.g.s. unit system.

$A$ : Numerical constant concerning the size distribution of large sea-salt nuclei. For example, $A=1.15 \times 10^{-12} \mathrm{~g} / \mathrm{cm}^{3}$ for nuclei measured in the days with surface wind force 4 .

$a=\frac{\rho_{w} r_{0}^{5}}{5 D K \rho_{\infty}}=5.74 \times 10^{-8} \mathrm{~g} \cdot \mathrm{sec}$

$b=\frac{4 \rho_{w}}{E M \alpha}=500 \mathrm{sec} \quad$ for $\quad M=1 \mathrm{~g} / \mathrm{m}^{3}$.

$C(t)$ : Salinity of rain water at the time $t$. Unit in $\mathrm{g} / \mathrm{cm}^{3}$.

$c=\frac{E M}{4 \rho_{w} r_{0}}=10^{-4} \mathrm{~cm}^{-1} \quad$ for $\quad M=1 \mathrm{~g} / \mathrm{m}^{3}$.

$c(r)$ : Salinity of rainrop in $\mathrm{g} / \mathrm{cm}^{3}$.

$D=0.25 \mathrm{~cm}^{2} / \mathrm{sec}$ : Diffusion coefficient of water vapour.

$D^{\prime}=D\left\{1+D L\left(\frac{\partial \rho_{0}}{\partial T}\right) / D_{T}\right\}^{-1}=0.42 D$ $=0.105 \mathrm{~cm}^{2} / \mathrm{sec}$ at $T=10^{\circ} \mathrm{C}$, where $L$ is latent heat of condensation, $D_{T}$ the thermal conductivity of air.

$E=1$ : Collection efficiency.

$E_{L}$ : Langmuir's collection efficiency.

$F(z, t)$ : Number of nuclei which reach the cloud base within time $t$ from the initial level higher than $z$. Unit in $\mathrm{cm}^{-2}$.

$f(\nu)$ : Size distribution of large sea-salt nuclei at $t=0$ expressed as a function of mass of nucleus. Unit in $\mathrm{cm}^{-3} \cdot \mathrm{g}^{-1}$.

$G(z)$ : Mass flux of liquid water at level $z$ in $\mathrm{g} / \mathrm{cm}^{2} / \mathrm{sec}$.

$G=-G(h)$ : Rate of outward transport of liquid water due to upper mixing. Unit in $\mathrm{g} / \mathrm{cm}^{2} / \mathrm{sec}$.

$g(r, t)$ : Size spectrum of rainfall flux. Unit in $\mathrm{cm}^{2} \cdot \mathrm{sec}^{-1}$.

$h$ : Cloud thickness in $\mathrm{cm}$.
$K=\frac{3}{4 \pi} k=0.136 \mathrm{~cm}^{3} / \mathrm{g}$.

$k=0.57 \mathrm{~cm}^{3} / \mathrm{g}$ : Raoult's constant for $\mathrm{NaCl}$ (Corresponding degree of electrolytic dissociation of $\mathrm{NaCl}$ is 0.84 )

$M$ : Liquid water content of the cloud. For example $M=1 \mathrm{~g} / \mathrm{m}^{3}=1 \times 10^{-6} \mathrm{~g} / \mathrm{cm}^{3}$.

$\bar{M}$ : Space average of $M$ between the base to the top of the cloud column.

$m$ : Rate of condensation in $\mathrm{g} / \mathrm{cm}^{3} / \mathrm{sec}$.

$\bar{m}$ : Space average of $\bar{m}$. For example $m$ $=1.91 \times 10^{-9} \mathrm{~g} / \mathrm{cm}^{3} / \mathrm{sec}$ for upward velocity $=1 \mathrm{~m} / \mathrm{sec}$.

$m^{\prime}$ : Apparent rate of condensation where effect of upper mixing is taken into consideration. Unit in $\mathrm{g} / \mathrm{cm}^{3} / \mathrm{sec}$.

$\boldsymbol{N}(t)$ : Mass flux of salt substance in $\mathrm{g} / \mathrm{cm}^{2} / \mathrm{sec}$.

$\boldsymbol{N}(\nu)$ : Cumulative number concentration of nuclei with masses larger than $\nu$.

$r$ : Drop radius.

$r_{0}=25 \mu=2.5 \times 10^{-3} \mathrm{~cm}$ : Critical radius both for growth and falling of droplets.

$r_{00}$ : Initial radius of droplet before the normal condensation begins.

$r_{\max }$ : Radius of largest raindrop at the time $t$.

$S$ : Degree of supersaturation of water vapour. For example $S=1 \%=0.01$.

$s$ : Radius of nucleus.

$t$ : time after the beginning of condensation in sec.

$t^{*}=a / \nu_{\max }+b \ln (c h+1): \quad$ The time when the largest nuclei starting at the top of the cloud reach the base. For example, $t^{*}$ $=1530 \mathrm{sec}$ for $\nu_{\max }=10^{-8} \mathrm{~g}, \quad M=1 \mathrm{~g} / \mathrm{m}^{3}$, and $h=2000 \mathrm{~m}$.

$V(r)=\alpha r: \quad$ Fall velocity of droplet in $\mathrm{cm} / \mathrm{sec}$ assumed in the simplified calculation.

$v_{r}$ : Fall velocity of droplet obtained experimentally by Gunn and Kinzer. Unit in $\mathrm{cm} / \mathrm{sec}$.

$W(t)$ : Rainfall intensity in $\mathrm{cm} / \mathrm{sec}$.

$z$ : Vertical distance in $\mathrm{cm}$.

$\alpha=8 \times 10^{3} \mathrm{sec}^{-1}$ : Numerical constant concerning fall velocity of droplet.

$\nu$ : Mass of nucleus in $g$.

$\nu_{\max }$ : Mass of largest nuclei at $t=0$. Unit in $\mathrm{g}$.

$\nu_{1}$ : Lower limit of mass of nuclei which becoming raindrops, can fall significantly relative to the air at time $t$. Unit in $g$. 
$\nu_{2}$ : Upper limit of mass of nuclei which remain within cloud at time $t$. Unit in $g$.

$\nu(z, t)$ : Mass of nucleus which is initially suspended at a height $z$, then falls and reaches the base at $t=t$. Unit in $\mathrm{g}$.

$\rho_{w}=1 \mathrm{~g} / \mathrm{cm}^{3}$ : Density of liquid water.

$\rho_{0}=10 \mathrm{~g} / \mathrm{m}^{3}=10^{-5} \mathrm{~g} / \mathrm{cm}^{3}$ : Saturation vapour density over flat surface of pure water.

$\rho_{\infty}$ : Vapour density in ambient air. Unit in $\mathrm{g} / \mathrm{cm}^{3}$.

$\rho_{r}$ : Saturation vapour density at the surface of water droplet containing salt substance. Unit in $\mathrm{g} / \mathrm{cm}^{3}$.

\section{References}

1. East, T.W.R. (1957): An inherent precipitation mechanism in cumulus clouds. Quart. J.R. Met. Soc., 83, 61-76.

2. Howell, W. E. (1949): The growth of cloud drops in uniformly cooled air. J. Met., 6, 134-149.

3. Junge, C. E. (1953): Die Rolle der Aerosole und der gasförmigen Beimengungen der Luft im Spurenstoffhaushalt der Troposphäre. Tellus, 5, $1-26$.

4. (1954): The chemical composition of atmospheric aerosols, I: Measurements at
Round Hill field station June-July 1953. J. Met., 11, 323-333.

5. (1956): Recent investigations in air chemistry. Tellus, 8, 127-139.

6. Komabayasi, M. (1957): Some aspects of rain formation in warm cloud (I). Salinity of individual raindrops and other quantities concerning rainfall. J. Met. Soc. Japan, Ser. II, 35, 205-220.

7. Ludlam, F. H. (1951): The production of showers by the coalescence of cloud droplets. Quart. J.R. Met. Soc., 77, 402-417.

8. Ludlam, F. H. and Scorer, R. S. (1953): Convection in the atmosphere. Quart. J. R. Met. Soc., 79, 317-341.

9. Pearcey, T. and Hill, G. W. (1957): A theoretical estimate of the collection efficiencies of small droplets. Quart. J. R. Met. Soc., 83, 77-92.

10. Stommel, H. (1947): Entrainment of air into a cumulus cloud. J. Met., 4, 491-496.

11. Telford, J. W. (1955): A new aspect of coalescence theory. J. Met., 12, 436-444.

12. Turner, J. S. (1955): The salinity of rainfall as a function of drop size. Quart. J. R. Met. Soc., 81, 418-429.

13. Woodcock, A.H. (1953): Salt nuclei in marine air as a function of altitude and wind force. J. Met., 10, 362-371. 